\title{
Mối quan hệ giữa văn hóa doanh nghiệp, quản lý chất lượng toàn diện và hiệu quả dự án tại các công ty lắp máy khu vụ̣c miền Bắc
}

\section{The relationship between corporate culture, total quality management and project efficiency in Northern machinery installation company of LILAMA}

\author{
Bùi Thị Minh Thu ${ }^{1 *}$, Phan Thị Yến Lai ${ }^{1}$ \\ ${ }^{1}$ Trường Đại Học Nội vụ Hà Nội - Phân hiệu Quảng Nam, Việt Nam \\ "Tác giả liên hệ, Email: thubtmgv@gmail.com
}

\section{THÔNG TIN}

DOI: $10.46223 / \mathrm{HCMCOUJS}$. econ.vi.16.1.1386.2021

Ngày nhận: 10/04/2020

Ngày nhận lại: 28/04/2020

Duyệt đăng: 03/08/2020

Tù khóa:

hiệu quả dự án, quản trị chất lượng toàn diện, văn hóa doanh nghiệp, công ty lắp máy

\section{TÓM TẮT}

Sự mở rộng thị trường mới trong lĩnh vực lắp máy đã tạo nên sự phát triển vượt bậc của Tổng công ty lắp máy Việt Nam (LILAMA), trong đó có sự góp công của các công ty Lắp máy khu vực Miền Bắc. Bài nghiên cứu này khảo sát mối quan hệ giữa VHDN, quản trị chất lượng toàn diện và hiệu quả dự án tại các công ty lắp máy khu vực Miền Bắc nơi mà các dự án về lắp máy được trúng thầu với những hợp đồng có giá trị cao. Dữ liệu thu thập từ 283 lao động tại các công ty lắp máy khu vực Miền Bắc của Lilama được phân tích bằng cách sử dụng mô hình Sem. Kết quả cho thấy VHDN và quản lý chất lượng toàn diện có ảnh hưởng trực tiếp đến hiệu quả dự án, từ đó đề ra những biện pháp về công tác quản trị tạo lợi thế cạnh tranh cho công ty lắp máy khu vực Miền Bắc của Lilama và ứng dụng các doanh nghiệp khác tại Việt Nam.

\section{ABSTRACT}

The expansion of the new market in the field of machine installation has made a great development of LILAMA, this includes the contribution of the Northern Installation Companies. This paper examines the relationship between corporate culture, Total Quality Management and project efficiency in Northern Machinery Installation Company of LILAMA where contractor projects have been awarded contracts with high-value contracts. Data collected from 283 employees of Northern Machinery Installation Company by LILAMA were analyzed by using the Sem model. The results showed that corporate culture and Total Quality Management have a direct impact on the project's effectiveness, thus setting out management measures that create a competitive advantage for the Northern machinery Installation Company of LILAMA and apply to other enterprises in Vietnam. project performance, total quality management, corporate culture, machine installation company 


\section{Giới thiệu}

Quá trình toàn cầu hóa và hội nhập kinh tế làm cho môi trường kinh doanh thay đổi một cách nhanh chóng. Để thành công, các doanh nghiệp phải thích ứng được với sự biến đổi của thị trường và tạo ra những lợi thế cạnh tranh bền vững (Barney, 2001). Nghiên cứu của Noe (2013), VHDN ngày nay được xem là một phần trong vốn xã hội tạo nên tài sản vô hình quyết định sự phát triển bền vững của doanh nghiệp. VHDN xác lập một hệ thống các giá trị được mọi người làm trong doanh nghiệp chia sẻ, chấp nhận, đề cao và ứng xử theo các giá trị đó. Qua đó, VHDN góp phần tạo nên sự khác biệt giữa các doanh nghiệp và được coi là nguồn của lợi thế cạnh tranh. Trong khi đó các công ty lắp máy khu vực Miền Bắc với hàng ngàn người lao động là một tập hợp những con người khác nhau về trình độ chuyên môn, trình độ văn hóa, mức độ nhận thức, quan hệ xã hội, vùng miền địa lý, tư tưởng văn hóa...chính sự khác nhau này tạo ra một môi trường làm việc đa dạng và phức tạp. Bên cạnh đó do tính chất ngành cơ khí nên các công ty Lắp máy khu vực Miền Bắc có môi trường làm việc nhanh, nhịp độ làm việc cao, thời gian làm việc dài, điều kiện làm việc khắc nghiệt ở nơi vùng sâu, vùng xa, trong quá trình làm có sự phụ thuộc lẫn nhau giữa các nhóm làm việc, vì thế cần xây dựng nền VHDN để người lao động hòa nhập, phát triển doanh nghiệp hơn.

Những nghiên cức hiện hành chỉ ra rằng có các nhân tố đóng vai trò thúc đẩy hiệu quả dự án. Kendra và Taplin (2004) đã chỉ ra rằng sự thành công dự án có bốn nhân tố: năng lực quản lý dự án, hệ thống đo lường, quy trình kinh doanh và cơ cấu tổ chức dự án. Có rất nhiều nghiên cứu chứng minh sự thành công của dự án bị ảnh hưởng bởi nhiều nhân tố như: Nghiên cứu của Parolia, Kundabala, Dahal, Mohan, và Thomas (2001) chỉ ra đó là sự phối hợp hiệu quả và chỉ ra đó là quản trị chất lượng toàn diện. Nghiên cứu của Kendra và Taplin (2004) đó là VHDN. Cummings và Worley (1997, p. 93) định nghĩa: "VHDN là tiềm lực để thúc đẩy sự liên kết giữa những nhiệm vụ khác nhau, để phục vụ cho mục tiêu của tổ chức và phát triển của nhân viên, thiết lập phương pháp để truyền tải thông tin xuyên suốt tổ chức". Parolia và cộng sự (2011) cho quản trị chất lượng toàn diện là làm cho toàn bộ tổ chức hoạt động với mục tiêu chất lượng của sản phẩm, đưa chất lượng vào sản phẩm và đánh giá sản phẩm để đạt được yêu cầu về chất lượng và sự lòng của khách hàng. Tuy nhiên, việc nghiên cứu tổng quản trị chất lượng và hiệu quả dự án trong môi trường văn hóa thì hiếm xảy ra. Câu hỏi của việc nghiên cứu là xác định mối liên hệ giữa VHDN, quản trị chất lượng toàn diện và hiệu quả dự án tại các công ty Lắp máy khu vực Miền Bắc. Đặc biệt, nghiên cứu muốn tìm ra mức độ quan trọng của quản trị chất lượng toàn diện đối với hiệu quả của dự án.

Vì thế tác giả chọn đề tài: "Mối quan hệ giữa VHDN, quản trị chất luợng toàn diện và hiệu quả dự án tại các công ty Lắp máy khu vực Miền Bắc”, để nghiên cứu tìm ra biện pháp về công tác quản trị tạo lợi thế cạnh tranh cho công ty lắp máy khu vực Miền Bắc của Lilama và ứng dụng các doanh nghiệp khác tại Việt Nam.

\section{Tổng quan tài liệu và phương pháp nghiên cứu}

\subsection{Khái niệm chính}

\subsubsection{Văn hóa doanh nghiệp}

Deal và Kennedy (1982) xác định VHDN bằng các giá trị: anh hùng, lễ nghi và nghi thức, các mạng lưới truyền thông. Cameron và Quinn (2006) đề xuất một khuôn khổ VHDN được đo lường dựa trên 39 chỉ số hiệu quả tổ chức. Một cách tiếp cận khác để xác định $\mathrm{OC}$ được dựa trên đặc điểm: tham gia, thống nhất, nhiệm vụ và khả năng thích ứng, phát triển bởi Kotrba (2010). Mô hình Denison mô hình này đã đưa ra các thang đo hay tiêu chí để đánh giá sự mạnh hay yếu của VHDN với 4 đặc điểm văn hoá (khả năng thích ứng, sứ mệnh, tính nhất quán, sự 
tham gia), trong mỗi đặc điểm có 3 cách thức biểu hiện và sử dụng 2 chiều: Tập trung bên trong so với tập trung bên ngoài, linh động so với ổn định. Theo Herzka và Turáková (2010), cho rằng mô hình đánh giá VHDN của Daniel R. Denison là lý tưởng nhất. Mô hình này đã được 5.000 DN và nhiều nhà nghiên cứu áp dụng trong hơn 20 năm qua.

\subsubsection{Quản trị chất lượng toàn diện}

Hendricks và Singhal (2001a) chỉ ra rằng việc ứng dụng kết quả quản trị chất lượng toàn diện (TQM) trong cải thiện hiệu quả tài chính và TQM đảm bảo chất lượng sản phẩm cao hơn. Demirbag, Tatoglu, Tekinkus, và Zaim (2006) đề nghị bảy yếu tố để đánh giá việc thực hiện TQM, cụ thể là: chất lượng số liệu và báo cáo, vai trò của quản lý; nhân viên quan hệ, quản lý chất lượng nhà cung cấp, đào tạo, chất lượng chính sách và quy trình quản lý. Vì vậy, dựa trên các nghiên cứu trên, nghiên cứu này đã sử dụng mô hình sửa đổi một số thành phần, bao gồm khả năng lãnh đạo, quản lý nguồn nhân lực, quy trình quản lý, quản lý hợp tác và liên tục cải tiến chất lượng và thông tin.

\subsubsection{Hiệu quả của dụ án (Project performance_PP)}

Thách thức lớn nhất của các nhà quản lý trong quản lý và tổ chức dự án là sự phức tap và không thể đoán trước của các dự án. Nghiên cứu của Kaplan và Norton (1996) đưa ra bảng điểm cân bằng (BSC) để quản lý dự án bao gồm: Khách hàng, tài chính, quy trình kinh doanh, đổi mới và tăng trưởng trong tổ chức. Trong việc đánh giá tác động của khả năng năng động $\mathrm{PP}, \mathrm{Wu}$, Tsai, Cheng, và Lai (2006) đã kiểm tra PP theo sáu cấp độ: phân tích chi phí, phân tích lợi ích, tỷ lệ thành công, chất lượng sản phẩm, cải tiến quy trình và đổi mới công nghệ. Dựa trên nghiên cứu tài liệu trên, hiện nay nghiên cứu sử dụng năm yếu tố, cụ thể là phân tích chi phí / lợi ích, tỷ lệ thành công, chất lượng sản phẩm, cải tiến quy trình và đổi mới công nghệ để đo lường $\mathrm{PP}$.

\subsection{Nhũng công trình nghiên cúu có liên quan}

Những nghiên cức hiện hành chỉ ra rằng có các nhân tố đóng vai trò thúc đẩy hiệu quả dự án. Kendra và Taplin (2004) đã chỉ ra rằng sự thành công dự án có bốn nhân tố: năng lực quản lý dự án, hệ thống đo lường, quy trình kinh doanh và cơ cấu tổ chức dự án. Có rất nhiều nghiên cứu chứng minh sự thành công của dự án bị ảnh hưởng bởi nhiều nhân tố như: Nghiên cứu của Parolia và cộng sự (2001) chỉ ra đó là sự phối hợp hiệu quả, nghiên cứu của Bryde và Ronison (2007) chỉ ra đó là quản trị chất lượng toàn diện, nghiên cứu của Kendra và Taplin (2004) đó là VHDN. Cummings và Worley (1997, p. 93) định nghĩa: "VHDN là tiềm lực để thúc đẩy sự liên kết giữa những nhiệm vụ khác nhau, để phục vụ cho mục tiêu của tổ chức và phát triển của nhân viên, thiết lập phương pháp để truyền tải thông tin xuyên suốt tổ chức". Parolia và cộng sự (2011) cho quản trị chất lượng toàn diện là làm cho toàn bộ tổ chức hoạt động với mục tiêu chất lượng của sản phẩm, đưa chất lượng vào sản phẩm và đánh giá sản phẩm để đạt được yêu cầu về chất lượng và sự lòng của khách hàng.

\subsection{Mô hình nghiên cúu}

Dựa theo các giả thiết nghiên cứu và tổng quan tài liệu, mô hình nghiên cứu được đề xuất:

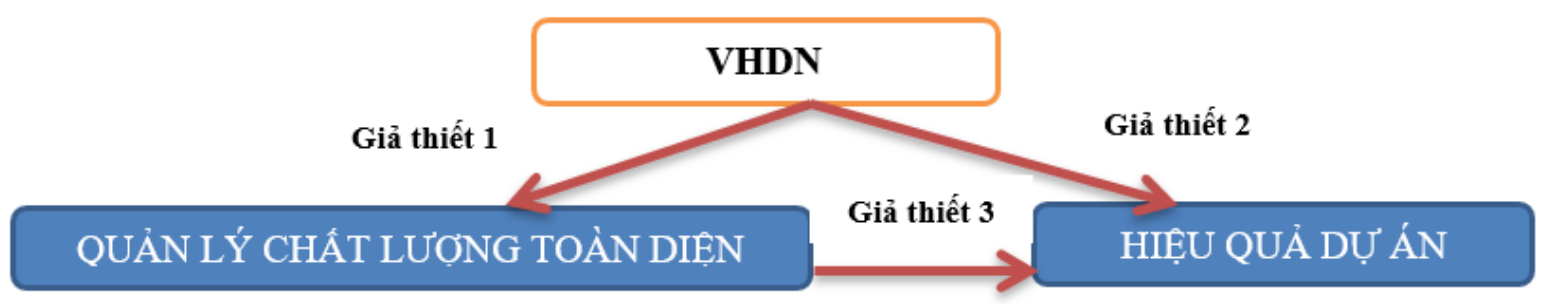

Hình 1. Mô hình nghiên cứu đề xuất 


\section{Phương pháp nghiên cứu}

\subsection{Giới thiệu nghiên cúu}

Để xây dựng câu hỏi khảo sát thì nhóm các chuyên gia bao gồm có 5 thầy cô với trình dộ nghiên cứu sâu về quản trị nhân lực ở Đại học Kinh tế Đà Nẵng, Đại học kinh tế Huế, 3 giám đốc Lilama đã cung cấp các thông tin, dữ liệu để xác định các yếu tố hình bảng câu hỏi khảo sát về Mối quan hệ giữa VHDN, quản trị chất lượng toàn diện và hiệu quả dự án tại các công ty Lắp máy khu vực Miền Bắc. Trong phần nghiên cứu định tính này, một số tên gọi đã được các chuyên gia góp ý để chỉnh sửa cho phù hợp với đặc điểm và điều kiện của công ty Lắp máy khu vực Miền Bắc. Để phân tích mối quan hệ giữa VHDN, quản trị chất lượng toàn diện và hiệu quả dự án tại các công ty Lắp máy khu vực Miền Bắc, nghiên cứu sử dụng kỹ thuật phân tích được xây dựng dựa trên nền tảng lý thuyết mô hình phương trình cấu trúc SEM (Structural Equation Modeling) và sự hỗ trợ của phần mềm SPSS 22 và phần mềm AMOS (Analysis Of Moment Structures). Với kỹ thuật phân tích này bỏ qua đa cộng tuyến và sự tin cậy của dữ liệu thị trường cũng được xem xét thông qua các sai số đo lường. Đánh giá độ tin cậy thang đo thông qua đại lượng Cronbach's Alpha, phân tích nhân tố khám phá EFA (Exploratory Factor Analysis). Kỹ thuật phân tích nhân tố khẳng định CFA, Kỹ thuật phân tích mô hình cấu trúc SEM.

\subsection{Phuơng pháp thu thập số liệu}

\subsubsection{Xác định cõ mẫu}

Theo các nhà nghiên cứu Hair, Anderson, Tatham, và Black (1998), thì để chọn kích thước quan sát nghiên cứu phù hợp đối với phân tích nhân tố khám phá EFA cỡ quan sát tối thiểu $\mathrm{N}>5^{*} \mathrm{x}$ ( $\mathrm{x}$ : là tồng số biến quan sát). Theo Tabachnick và Fideel (1996) để tiến hành phân tích hồi quy của một cách tốt nhất thì cỡ quan sát tối thiểu cần đạt được tính theo công thức $\mathrm{N}>$ $50+8 \mathrm{~m}$ (trong đó $\mathrm{m}$ là biến độc lập). Phù hợp với nghiên cứu của mình thì tác giả sử dụng số quan sát ứng với 4 nhóm và 12 biến của VHDN, 5 biến của quản trị chất lượng toàn diện, 4 biến của hiệu quả dự án là : $N>\max (5 \times 12 ; 50+9 \times 8)=(60,122)=122$ quan sát. Do các công ty Lắp máy khu vực Miền Bắc có số lượng nhân viên đông nên tác giả lấy 300 số liệu quan sát để đảm bảo tính chính xác hơn cho dữ liệu nghiên cứu.

\subsubsection{Phuoong pháp chọn mẫu}

Việc lựa chọn phương pháp chọn quan sát ở đây là phương pháp chọn quan sát theo hạn ngạch (quota) của các nhóm đối tượng được phân chia theo biến số địa bàn, khu vực. Đối tượng tham gia trong nghiên cứu này bao gồm người lao động của các công ty Lắp máy khu vực Miền Bắc: Công ty cổ phần Lilama 69.1 và 69.2, Công ty Cố phẩn Lilama 5 , tổng cộng 300 quan sát phát ra, thu vào là 283 quan sát. Việc phỏng vấn đáp viên theo hình thức phát trực tiếp tại nơi làm việc là 98 phiếu, gửi mail là 205 phiếu.

\subsection{Giả thiết nghiên cúu}

\subsubsection{Mối quan hệ giũa văn hóa doanh nghiệp và quản lý chất luợng toàn diện}

Nhiều nghiên cứu chứng minh sự thành công của thực hiện quản lý chất lượng toàn diện phần lớn phụ thuộc vào VHDN. Mosadegh (2006) đã xác định tác động của các giá trị VHDN đối với sự thành công của việc thực hiện quản lý chất lượng toàn diện tại một bệnh viện ở Iran. Kết quả cho thấy rằng quản lý chất lượng toàn diện đòi hỏi một nền VHDN được tổ chức theo định hướng chất lượng, tổ chức học tập, tinh thần kinh doanh, làm việc nhóm và sự hợp tác, chấp nhận rủi ro, liên tục cải tiến, tập trung khách hàng, quan hệ đối tác với các nhà cung cấp, theo dõi và đánh giá chất lượng. Prajogo và $\mathrm{MCDermott} \mathrm{(2005)} \mathrm{đã} \mathrm{phát} \mathrm{hiện} \mathrm{ra} \mathrm{rằng} \mathrm{sự} \mathrm{khác} \mathrm{nhau} \mathrm{của}$ việc thực hiện quản lý chất lượng toàn diện được xác định bởi sự khác nhau của các nền VHDN. Đặc biệt, các nền VHDN có phân chia thứ bậc đã chỉ ra được mối quan hệ đáng kể với thực tiê̂n 
của việc quản trị chất lượng toàn diện. Theo các nghiên cứu đã trình bày ở trên, nghiên cứu này đề xuất giả thuyết sau đây:

\section{H1: Văn hóa doanh nghiệp ảnh hwởng tích cực đến quản lý chất lượng toàn diện}

\subsubsection{Mối quan hệ giũa văn hóa doanh nghiệp và hiệu quả dụ án}

Jabnoun và Sedrani (2005) thấy rằng những tác động kết hợp của VHDN và quản lý chất lượng toàn diện thì ảnh hưởng đáng kể tới hiệu quả dự án. Dulaimi, Nepal, và Park (2005) đã cho rằng nhà quản lý cấp cao nên cung cấp nguồn lực và hỗ trợ để tạo ra một môi trường hay văn hóa nhằm tạo điều kiện cho người quản lý dự án trong việc thực hiện dự án. Như vậy có mối quan hệ giữa VHDN và hiệu quả dự án, giả thuyết nghiên cứu như sau:

\section{H2: Văn hóa doanh nghiệp ảnh hương tích cực đến hiệu quả dụ án}

\subsubsection{Mối quan hệ giũa quản lý chất lương toàn diện và hiệu quả dự án}

Trong nghiên cứu mối quan hệ giữa quản lý chất lượng toàn diện và hiệu quả dự án, Prasad, Rajan, và Subranmanian (2007) đã phát hiện ra giữa 2 vấn đề này có mối quan hệ với nhau. Cải thiện hiệu quả dự án là mong muốn của mỗi công ty. Bryde và Robinson (2007) đã tìm ra ảnh hưởng của quản lý chất lượng toàn diện ở mức độ cao trong hoạt động quản lý dự án và cũng thấy được quản lý chất lượng toàn diện đã cải thiện được hiệu quả dự án thông qua việc coi trọng đáp ứng nhu cầu khách hàng. Vì vậy, chúng tôi đưa ra giả thuyết rằng:

\section{H3: Quản lý chất lượng toàn diện có ảnh hương tích cực đến hiệu quả dư án}

\subsection{Lụa chọn và phát triển thang đo}

Bảng câu hỏi bao gồm bốn phần: văn hóa doanh nghiệp, quản lý chất lượng toàn diện, hiệu quả dự án và nền tảng cá nhân được trả lời bằng cách sử dụng thang đo Liker 5 điểm sau:

\subsubsection{Văn hóa doanh nghiệp:}

Dựa trên mô hình (2010) của Kotrba, bốn cấu trúc lớn được xem xét:

(1) Sự tham gia: đề cập đến mức độ mà tổ chức này tập trung vào việc phát triển, thông báo, liên quan đến người dân và nhận được sự tham gia từ họ, liên quan đến nhân viên đó là khả năng, quyền sở hữu và trách nhiệm;

(2) Tính nhất quán: đề cập đến mức độ mà tổ chức này có một mạnh mẽ và gắn kết nội bộ, văn hóa, liên quan đến những giá trị chung, cách để hòa giải những bất đồng, phối hợp và tích hợp giữa các đơn vị chức năng khác nhau;

(3) Nhiệm vụ: đề cập đến mức độ mà tổ chức này có một ý thức rõ ràng về mục đích định hướng lâu dài, bao gồm cả tầm nhìn, chỉ đạo chiến lược, mục đích và mục tiêu;

(4) Khả năng thích nghi: mức độ mà khả năng thích ứng của tổ chức một cách nhanh chóng với những tín hiệu từ môi trường bên ngoài, bao gồm cả khách hàng và thị trường.

\section{Bảng 1}

Bảng tóm tắt câu hỏi văn hóa doanh nghiệp

\begin{tabular}{|l|c|}
\hline Kích thước & Yếu tố \\
\hline Văn hóa doanh nghiệp & Sự tham gia \\
\cline { 2 - 3 } & Sự nhất quán \\
\cline { 2 - 3 } & Khả năng thích nghi \\
\cline { 2 - 2 } & Sứ mệnh \\
\hline
\end{tabular}

Nguồn: Số liệu điều tra khảo sát của tác giả năm 2019 


\subsubsection{Quản lý chất luợng toàn diện}

Dựa trên các tài liệu (Demirbag et al., 2006; Hendricks \& Singhal, 2001a), quản trị chất lượng toàn diện đã được đo lường bằng:

(1) Khả năng lãnh đạo: Là việc nhà quản lý tạo ra một môi trường làm việc trong đó nhân viên tôn trọng lẫn nhau, thông tin được cung cấp đầy đủ, được tham gia vào quá trình thực hiện kinh doanh để nâng cao việc quản lý chất lượng toàn diện;

(2) Quản lý nguồn nhân lực: là hệ thống các triết lý, chính sách và hoạt động chức năng về thu hút, đào tạo - phát triển và duy trì con người của một tổ chức nhằm đạt được kết quả tối ưu cho cả tổ chức và nhân viên;

(3) Quy trình quản lý: đề cập đến mức độ mà công ty trao quyền cho lãnh đạo dự án hoặc các chuyên gia để quản lý thực hiện dự án trong quá trình lập kế hoạch và thiết kế dự án;

(4) Mối quan hệ hợp tác: đề cập đến mức độ đo lường mối quan hệ hợp tác với các liên minh bên ngoài, về trách nhiệm nâng cao chất lượng, các kênh truyền thông, cũng như cung cấp các hướng dẫn rõ ràng về yêu cầu chất lượng để hợp tác với các công ty bên ngoài;

(5) Liên tục cải thiện chất lượng: đề cập đến các chiến lược của công ty trong việc liên tục cải tiến chất lượng.

\section{Bảng 2}

Bảng tóm tắt câu hỏi quản lý chất lượng toàn diện

\begin{tabular}{|c|c|}
\hline Kích thước & Yếu tố \\
\hline Quản lý chất lương toàn diện & Quản lý nguồn nhân lực \\
\cline { 2 - 3 } & Khả năng lãnh đạo \\
\cline { 2 - 3 } & Quy trình quản lý \\
\cline { 2 - 3 } & Cải thiện thông tin chất lượng \\
\cline { 2 - 3 } & Quản lý sự hợp tác doanh nghiệp \\
\hline
\end{tabular}

Nguồn: Số liệu điều tra khảo sát của tác giả năm 2019

\subsubsection{Hiệu quả dụ án}

Wu và cộng sự (2006) đã đưa ra 5 cấp độ của hiệu quả dự án:

(1) Hiệu suất (chi phí /lợi ích) dự án là mối quan hệ giữa: chi phí là đề cập đến thỏa thuận tài chính đối với các dự án và lợi ích dự án là kết quả đáp ứng của dự án;

(2) Kiểm soát tỷ lệ thành công dự án: Liên quan đến việc thiết lập các hệ thống và quy trình ra quyết định để quản lý sự khác biệt giữa kế hoạch (phạm vi, chi phí, tiến độ.) và thực tế thực hiện dự án;

(3) Chất lượng sản phẩm: đề cập đến chất lượng của dự án cốt lõi, chức năng sản phẩm, và chức năng lợi ích cho khách hàng;

(4) Cải tiến qui trình đề cập đến quá trình dự án, và mức độ mà mới ý tưởng liên tục được tạo ra để hỗ trợ thực hiện dự án dựa trên việc khảo sát thị trường;

(5) Công nghệ và đổi mới: đề cập đến mức độ mà tổ chức tích hợp bước đột phá công nghệ mới lạ và cải tiến nhỏ hỗ trợ thực hiện dự án. 


\section{Bảng 3}

Bảng tóm tắt câu hỏi hiệu quả dự án

\begin{tabular}{|l|c|}
\hline \multicolumn{1}{|c|}{ Kích thước } & Yếu tố \\
\hline Hiệu quả dự án & Đổi mới công nghệ \\
\cline { 2 - 3 } & Tỷ lệ thành công \\
\cline { 2 - 3 } & Phân tích chi phí / lợi ích \\
\cline { 2 - 3 } & Chất lượng sản phẩm \\
\hline & Cải tiến qui trình \\
\hline
\end{tabular}

Nguồn: Số liệu điều tra khảo sát của tác giả năm 2019

\section{Kết quả và thảo luận}

\section{Bảng 4}

Số lượng và tiêu chí người tham gia khảo sát

\begin{tabular}{|l|l|c|c|}
\hline \multicolumn{2}{|c|}{ Tiêu chí } & Số lượng (người) & Tỷ trọng (\%) \\
\hline \multirow{3}{*}{ Giới tính } & Nam & 238 & 84,10 \\
\cline { 2 - 4 } & Nữ & 45 & 15,90 \\
\hline \multirow{3}{*}{ Độ tuổi } & Dưới 35 & 165 & 58,30 \\
\cline { 2 - 4 } & Từ 35-50 & 88 & 31,10 \\
\cline { 2 - 4 } & Trên 50 & 30 & 10,60 \\
\hline \multirow{5}{*}{ Trình độ } & Dưới đại học & 142 & 50,18 \\
\cline { 2 - 4 } & Đại học & 140 & 49,47 \\
\cline { 2 - 4 } & Trên Đại học & 01 & 0,35 \\
\hline \multirow{3}{*}{ Thâm niên } & Dưới 5 năm & 138 & 48,76 \\
\cline { 2 - 4 } & Từ 5 - 10 năm & 82 & 28,98 \\
\cline { 2 - 4 } & Trên 10 năm & 63 & 22,26 \\
\hline \multirow{3}{*}{ Thu nhập } & Dưới 3 triệu & 14 & 4,95 \\
\cline { 2 - 4 } & Từ 3-10 triệu & 262 & 92,58 \\
\cline { 2 - 4 } & Trên 10 triệu & 9 & 2,47 \\
\hline
\end{tabular}

Nguồn: Số liệu điều tra khảo sát của tác giả năm 2019

Bảng 4 trình bày đặc điểm nhân khẩu học của mẫu, các dữ liệu được sử dụng trong nghiên cứu này bao gồm các câu trả lời từ những người lao động trong Công ty cổ phần Lilama 69.1 và 69.2, Công ty Cố phẩn Lilama 5. Mỗi công ty đã nhận được 110 phiếu để trả lời. Như vậy, tổng cộng 330 phiếu điều ra, thu vào được 283 phiếu (94.3\%), số liệu tương đối phù hợp. 


\section{Bảng 5}

Khảo sát thống kế cấu trúc và mô tả cho các chỉ số đo lường

\begin{tabular}{|l|c|c|c|c|}
\hline \multicolumn{1}{|c|}{ Chỉ số đo lường } & Mức ý nghĩa & Std.dev & Thứ tự & Cronbach's \\
\hline Văn hóa doanh nghiệp & 3,112 & 0,4815 & 3 & 0,8820 \\
\hline Quản lý chất lượng toàn diện & 3,371 & 0,3632 & 2 & 0,8123 \\
\hline Hiệu quả dự án & 3,522 & 0,3415 & 1 & 0,8948 \\
\hline
\end{tabular}

Nguồn: Số liệu điều tra khảo sát của tác giả năm 2019

Bảng 5 cho thấy các số liệu thống kê mô tả cho kích thước, kiểm tra độ tin cậy và tính hợp lệ, độ tin cậy và tính hợp lệ đã được tiến hành cho mỗi cấu trúc với các biện pháp đa biến. Hệ số và độ tin cậy ước tính đã được sử dụng để đo lường nội bộ.

\section{Kiểm tra mức độ tin cậy và giá trị hiệu lực}

Kiểm tra mức độ tin cậy và hiệu lực đã được thực hiện cho từng khái niệm với nhiều biện pháp đo lường các đa biến ngẫu nhiên. Ước chừng chỉ số mức tin cậy “Cronbach" thì được dùng để đo lượng tính nhất quán của các quy mô đa biến. Trong nghiên cứu này, một Cronbach của mỗi cấu trúc lớn hơn 0,8 , sẽ có độ tin cậy cho công cụ khảo sát. Ngoài ra, hệ số tương quan biến tổng lớn hơn 0,6 được coi là có giá trị tiêu chuẩn cao (Kerlinger \& Lee, 1999). Bảng 3 đã cho ra số liệu phù hợp với yêu cầu.

\section{Bảng 6}

Phân tích nhân tố và giá trị nhất quán nội bộ cho các câu hỏi

\begin{tabular}{|l|l|c|c|}
\hline \multicolumn{1}{|c|}{ Kích thước } & \multicolumn{1}{|c|}{ Yếu tố } & Tổng tương quan & Cronbach's \\
\hline \multirow{4}{*}{$\begin{array}{l}\text { Văn hóa doanh } \\
\text { nghiệp }\end{array}$} & Sự tham gia & 0,6004 & 0,9005 \\
\cline { 2 - 4 } & Sự nhất quán & 0,6009 & 0,8654 \\
\cline { 2 - 4 } & Khả năng thích nghi & 0,5982 & 0,8032 \\
\cline { 2 - 4 } & Sứ mệnh & 0,6124 & 0,8564 \\
\hline \multirow{4}{*}{$\begin{array}{l}\text { Quản lý chất } \\
\text { lương toàn } \\
\text { diện }\end{array}$} & Quản lý nguồn nhân lực & 0,6089 & 0,9123 \\
\cline { 2 - 4 } & Khả năng lãnh đạo & 0,5899 & 0,8850 \\
\cline { 2 - 4 } & Quy trình quản lý & 0,6045 & 0,8972 \\
\cline { 2 - 4 } & Cải thiện thông tin chất lượng & 0,5789 & 0,8154 \\
\cline { 2 - 4 } & Quản lý sự hợp tác doanh nghiệp & 0,5896 & 0,9005 \\
\hline \multirow{4}{*}{$\begin{array}{l}\text { Hiệu quả dự } \\
\text { án }\end{array}$} & Đổi mới công nghệ & 0,6023 & 0,9001 \\
\cline { 2 - 4 } & Tỷ lệ thành công & 0,5986 & 0,8523 \\
\cline { 2 - 4 } & Phân tích chi phí / lợi ích & 0,6054 & 0,8871 \\
\cline { 2 - 4 } & Chất lượng sản phẩm & 0,5689 & 0,8254 \\
\cline { 2 - 4 } & Cải tiến qui trình & 0,6096 & 0,8997 \\
\hline
\end{tabular}

Nguồn: Số liệu điều tra khảo sát của tác giả năm 2019 
Kết quả của phân tích nhân tố thăm dò được trình bày trong bảng 6 , phân tích nhân tố xác nhận trong đó bao gồm các giá trị hội tụ và phân biệt được thực hiện theo tiêu chuẩn. Kết quả cho thấy tất cả các mối tương quan lớn hơn không và đủ lớn để tiến hành có giá trị phân biệt. Hơn nữa, giá trị phân biệt đã được kiểm tra bằng cách đếm số lần một mục tương quan cao với các mục từ các yếu tố khác hơn so với các mặt hàng từ yếu tố riêng của mình. Các kết quả cũng cho thấy giá trị phân biệt đầy đủ. Trong nghiên cứu này thể hiện giá trị hội tụ và phân biệt.

\section{Bảng 7}

Quan sát chỉ số độ tin cậy của các yếu tố

\begin{tabular}{|l|l|c|}
\hline \multicolumn{1}{|c|}{ Kích thước } & \multicolumn{1}{|c|}{ Yếu tố } & Quan sát chỉ số tin cậy (R2) \\
\hline \multirow{4}{*}{$\begin{array}{l}\text { Văn hoá doanh } \\
\text { nghiệp }\end{array}$} & Sự tham gia & 0,61 \\
\cline { 2 - 3 } & Tính nhất quán & 0,67 \\
\cline { 2 - 3 } & Khả năng thích ứng & 0,65 \\
\cline { 2 - 3 } & Sứ mệnh & 0,62 \\
\hline \multirow{4}{*}{$\begin{array}{l}\text { Quản lý chất lương diện } \\
\text { toản }\end{array}$} & Quản lý nguồn nhân lực & 0,68 \\
\cline { 2 - 3 } & Khả năng lãnh đạo & 0,57 \\
\cline { 2 - 3 } & Quy trình quản lý & 0,56 \\
\cline { 2 - 3 } & Cải thiện và thông tin chất lượng liên tục & 0,62 \\
\cline { 2 - 3 } & Quản lý sự hợp tác của doanh nghiệp & 0,59 \\
\hline \multirow{4}{*}{ Hiệu quả dự án } & Đổi mới công nghệ & 0,65 \\
\cline { 2 - 3 } & Tỷ lệ thành công & 0,61 \\
\cline { 2 - 3 } & Phân tích chi phí / lợi ích & 0,52 \\
\cline { 2 - 3 } & Chất lượng sản phẩm & 0,58 \\
\cline { 2 - 3 } & Cải tiến qui trình & 0,59 \\
\hline
\end{tabular}

Nguồn: Số liệu điều tra khảo sát của tác giả năm 2019

\section{Mô hình SEM thể hiện mối quan hệ}

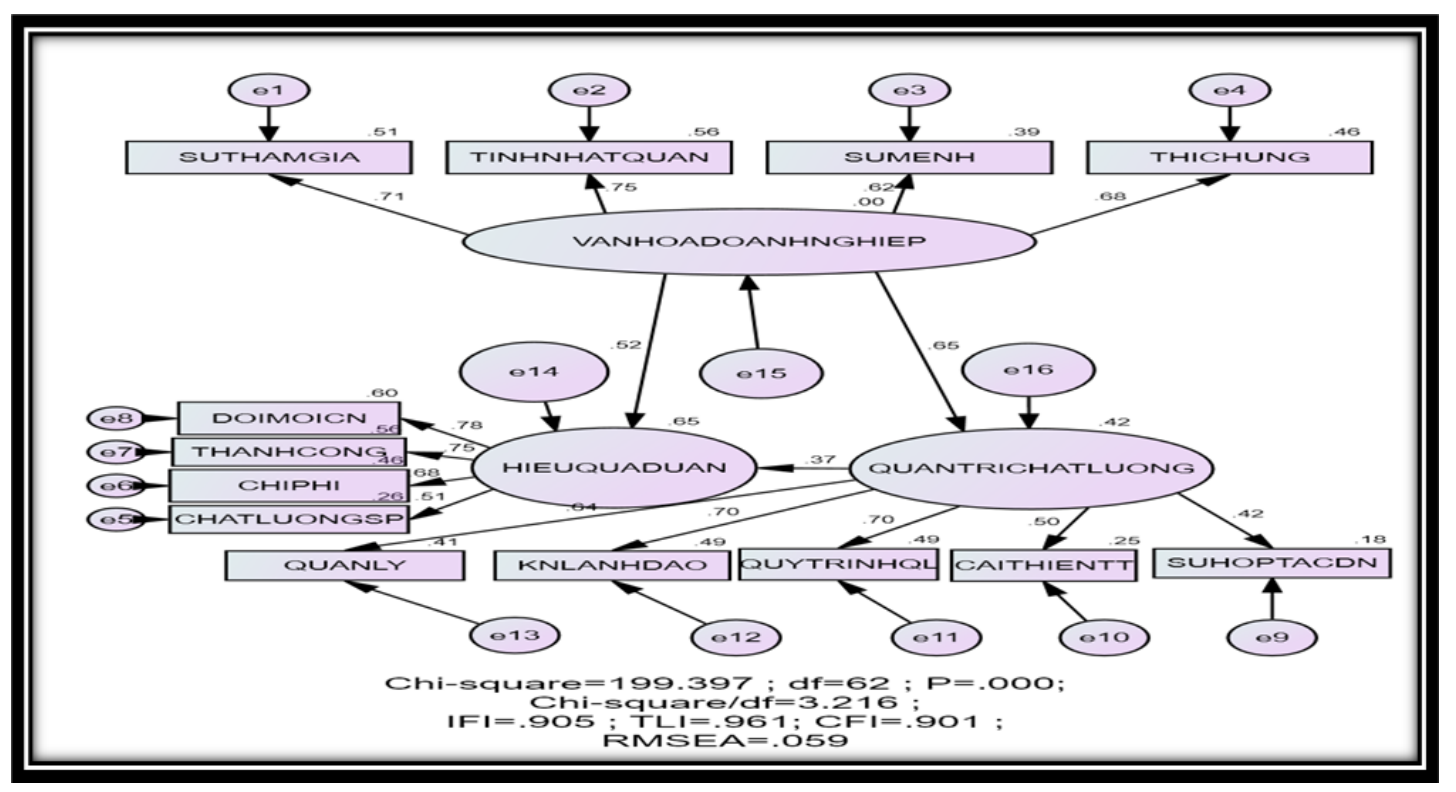

Hình 2. Mô hình SEM thể hiện mối quan hệ VHDN, quản trị chất lượng toàn diện và hiệu quả dự án Nguồn: Số liệu điều tra khảo sát của tác giả năm 2019 
Kết cấu mô hình phương trình đã được áp dụng để kiểm tra đề xuất các mô hình và giả thuyết. Mô hình phương trình cấu trúc (SEM) là một kỹ thuật thống kê đa biến để thử nghiệm lý thuyết cấu trúc. Trong mô hình đề xuất (Hình 1), VHDN được coi là một biến ngoại sinh, và hiệu quả dự án được xem là một biến nội sinh. Quản lý chất lượng toàn diện phục vụ cả hai như là một biến nội sinh (VHDN) nội sinh và biến ngoại sinh (hiệu quả dự án). Các câu hỏi cá nhân đã được tổng hợp thành các nhóm yếu tố cụ thể. Việc phân tích mô hình SEM được thể hiện trong hình 2 và phù hợp với các chỉ số tuyệt đối (IFI $=0,905, \mathrm{TLI}=0,961, \mathrm{CFI}=0,901, \mathrm{RMSEA}=$ 0,059 chỉ ra rằng các mô hình cấu trúc hoặc đáp ứng hoặc cao hơn giới hạn, và do đó đại diện cho một sự phù hợp thỏa đáng cho các dữ liệu mẫu thu thập được. Các số liệu thống kê chi bình phương chia cho độ tự do cũng chỉ ra một sự phù hợp, hợp lý Dựa trên hình 2 , cả ba mối quan hệ giả thuyết (H1, H2, và H3) cho thấy ý nghĩa thống kê (xem Bảng 5).

\section{Bảng 8}

Những quan sát được tổng kết lại từ phân tích mô hình

\begin{tabular}{|c|c|c|}
\hline Giả thuyết & Đường dẫn & Kết quả \\
\hline H1 & $\begin{array}{l}\text { Văn hóa doanh nghiệp (VHDN)-> Quản trị chất lượng toàn } \\
\text { diện }\end{array}$ & Có ý nghĩa thống kê \\
\hline $\mathbf{H 2}$ & Văn hóa doanh nghiệp -> hiệu quả dự án & Có ý nghĩa thống kê \\
\hline $\mathbf{H 3}$ & $\begin{array}{l}\text { Quản trị chất lượng toàn diện (TQM) -> hiệu quả dự án } \\
\text { (PP) }\end{array}$ & Có ý nghĩa thống kê \\
\hline
\end{tabular}

Nguồn: Số liệu điều tra khảo sát của tác giả năm 2019

\section{Thảo luận}

Dựa trên kết quả của phân tích, kết luận VHDN có ảnh hưởng tích cực trực tiếp lên TQM và $\mathrm{PP}(\mathrm{H} 1$ và $\mathrm{H} 2$ được ủng hộ). Thứ hai, $\mathrm{TQM}$ cũng có ảnh hưởng trực tiếp và tích cực đối với PP (H3 được ủng hộ). Thứ ba, những kết quả cũng thể hiện rằng sự ảnh hưởng gián tiếp của VHDN đối với PP $(\mathrm{H} 2)$.

Kết quả nghiên cứu hiện tại ủng hộ những phát hiện của những nghiên cứu trước đây liên quan đến ảnh hưởng của VHDN đối với TQM (Mosadegh, 2006). Ưng hộ cho kết luận này có thể được tìm thấy trong nhiều nghiên cứu của Dulaimi và cộng sự (2005), Jabnoun và Sedrani (2005). Trong nghiên cứu của họ, họ phát hiện ra rằng sự tương đồng trong VHDN của đối tác có một ảnh hưởng trực tiếp đáng kể đến quản lý chất lượng toàn diện lên hiệu quả dự án Do đó những nghiên cứu tồn tại ủng hộ $\mathrm{H} 1$ và $\mathrm{H} 2$, kết luận rằng $\mathrm{VHDN}$ đóng một vai trò quan trọng trong phát triển $\mathrm{TQM}$ và $\mathrm{PP}$ trong doanh nghiệp. Cuối cùng, nghiên cứu kết luận rằng $\mathrm{TQM}$ có một ảnh hưởng tích cực lên PP. Bên cạnh đó, nó tăng cường ảnh hưởng của VHDN đối với PP. Những nghiên cứu chứng minh ảnh hưởng sâu sắc của TQM đến hiệu quả dự án (Prasad et al., 2007), và nghiên cứu của Arditi và Lee (2003) cũng khẳng định điều này. Do đó, kết luận TQM đóng vai trò quan trọng trong việc nâng cao hiệu quả dự án của doanh nghiệp.

\section{Kết luận}

Kết quả nghiên cứu của tác giả đã chứng minh có mối quan hệ giữa VHDN, quản trị chất lượng toàn diện và hiệu quả dự án. Điều này hàm ý rằng nếu một công ty muốn thành công trên thị trường thì cần quan tâm đến văn hóa doanh nghiệp, thúc đẩy và phát triển văn hóa doanh nghiệp cũng là thúc đẩy tình hình phát triển của công ty. Đặc biệt là với các công ty cơ khí cần nhất là hiệu quả các dự án được hoàn thành một cách có hiệu quả và quản lý được chất lượng 
toàn diện. Cuối cùng, mặc dù các kết quả thực nghiệm từ nghiên cứu này chủ yếu hỗ trợ cho mô hình hiện tại, có ít nhất 2 hạn chế cần được xem xét kỹ lưỡng. Thứ nhất, bởi vì tính cá nhân trong việc cung cấp dữ liệu thực nghiệm, những thành kiến cũng như sở thích có thể tồn tại do sự khác nhau về kinh nghiệm cá nhân hoặc quá trình đào tạo. Thứ 2 , dữ liệu được thu thập tại Công ty cổ phần Lilama 69.1 và 69.2, Công ty Cố phẩn Lilama 5; những đặc tính của các công ty được điều tra có thể khác biệt với các công ty từ các khu vực hay quốc gia khác. Do đó, kết quả hiện tại không nên được giả định để đại diện cho trường hợp tổng quát. Tuy nhiên, chúng có thể cung cấp một tài liệu tham khảo cơ bản cho các công ty nằm trong những khu vực có môi trường tương tự như các Công ty cổ phần Lilama 69.1 và 69.2 , Công ty Cố phẩn Lilama 5.

\section{Tài liệu tham khảo}

Arditi, D., \& Lee, D.-E. (2003) Assessing the corporate service quality performance of design build contractors using quality function deployment. Construction Management and Economics, 21(2), 175-185.

Barney, J. B. (2001). Is the resource-based view a useful perspective for strategic management research? Yes. Academy of Management Review, 26(1), 41-54.

Bryde, D. J., \& Robinson, L. (2007). The relationship between total quality management and the focus of project management practices. The TQM Magazine, 19(1), 50-61.

Cameron, K., \& Quinn, R. E. (2006). Diagnosing and changing organizational culture: Based on the competing values framework. Beijing, China: China Renmin University Press.

Cummings, T. G., \& Worley, C. G. (1997). Organization development and change. Cincinnati, $\mathrm{OH}$ : Southwestern.

Curkovic, S., Vickery, S. K., \& Droge, C. (2000). An empirical analysis of the competitive dimensions of quality performance in the automotive supply industry. International Journal of Operations \& Production Management, 20(3), 386-403.

Deal, T. W., \& Kennedy, A. A. (1982). Corporate cultures. Reading, MA: Addison-Wesley.

Demirbag, M., Tatoglu, E., Tekinkus, M., \& Zaim, S. (2006). An analysis of the relationship between total quality management implementation and organizational performance Turkish SMEs. Journal of Manufacturing Technology Management, 17(6), 829-847.

Dulaimi, M. F., Nepal, M. P., \& Park, M. (2005). A hierarchical structural model of assessing innovation and project performance. Construction Management and Economics, 23(6), 565-577.

Hair, J. F., Jr., Anderson, R. E., Tatham, R. L., \& Black, W. C. (1998). Multivariate data analysis (5th ed.). New York, NY: Macmillan Publishing Company.

Hendricks, K. B., \& Singhal, V. R. (2001a). Firm characteristics, total quality management, and financial performance. Journal of Operations Management, 19(1), 269-285.

Herzka, P., \& Turáková, A. (2010). The applying denison model for the analysis of corporate. Retried January 5, 2020, from http://leidykla.vgtu.lt/conferences/BME_2010/0 06/004.htm

Jabnoun, N., \& Sedrani, K. (2005). TQM, culture, and performance in UAE manufacturing firms. Quality Management Journal, 12(4), 10-16. 
Kaplan, R. S., \& Norton, D. P. (1996). The balanced scorecard: Translating strategy into action. New York, NY: McGraw Hill.

Kendra, K., \& Taplin, L. J. (2004). Project success: A cultural framework. Project Management Journal, 35, 30-45.

Kerlinger, F. N., \& Lee, H. B. (1999). Investigación del comportamiento: Técnicas y. metodología. Mexico: Mc Graw Hill.

Koerniadi, H., \& Tourani-Rad, A. (2012). Does board independence matter? Evidence from New Zealand. Australasian Accounting, Business, and Finance Journal, 6(2), 3-18.

Kotrba, L. (2010), Organizational culture \& employee engagement: What's the relationship? Denison Consulting, 4(3), 415-419.

Mosadegh, R. A. (2006). A comparison of four models of performance management in healthcare systems. Information and Management in Health Biquarterly, 2(2), 71-78.

Noe, R. (2013). Employee training and development (6th ed.). New York, NY: McGraw Hill Education.

Northcraft, T., \& Neale, H. (1996). Organisation behaviour. London, UK: Prentice-Hall.

Parolia, A., Kundabala, M., Dahal, M., Mohan, M., \& Thomas, M. S. (2011). Management of supernumerary teeth. Journal of Conservative Dentistry, 14(3), 221-224.

Prajogo, D. I., \& McDermott, C. M. (2005). The relationship between total quality management practices and organizational culture. International Journal of Operations \& Production Management, 25(11), 1101-1122.

Prasad, E. S., Rajan, R. G., \& Subramanian, A. (2007). Foreign capital and economic growth. Brookings Papers on Economic Activity, Economic Studies Program, The Brookings Institution, 38(1), 153-230.

Saraph, J. V., Benson, P. G., \& Schroeder, R. G. (1989). An instrument for measuring the critical factors of quality management. Decision Sciences, 20, 810-829.

Tabachnick, B. G., \& Fidell, L. S. (1996). Using multivariate statistics (3rd ed.). New York, NY: Harpercollins College Div.

Wu, W. Y., Tsai, H. J., Cheng, K. Y., \& Lai, M. K. (2006). Assessment of intellectual capital management in Taiwanese IC design companies: Using dea and the malmquist productivity index. $R$ and D Management, 36(5), 531-545. 